\title{
Rates of blood cultures positive for vancomycin-resistant Enterococcus in Ontario: a quasi-experimental study
}

\author{
Jennie Johnstone MD PhD, Michelle E. Policarpio MSc, Freda Lam MPH, Kwaku Adomako MSc, \\ Chatura Prematunge MSc, Emily Nadolny MA MPH, Ye Li PhD, Kevin Brown PhD, \\ Elaine Kerr ART BA, Gary Garber MD
}

\section{Abstract}

Background: Some Ontario hospitals have discontinued active screening and isolation programs for vancomycin-resistant Enterococcus (VRE). The aim of this study was to determine whether this practice change is associated with a change in the rate of rise of VRE-positive blood cultures.

Methods: All Ontario hospitals are mandated to report VRE bacteremia. Using this publicly reported data set, we included all validated results between January 2009 and June 2015. Beginning in June 2012, some hospitals discontinued active VRE screening and isolation programs (intervention). We used an interrupted time series Poisson regression to assess the slope change in the incidence rate of VRE-positive blood cultures (primary outcome) after versus before the intervention. Hospitals that continued to screen were the comparison group. Incidence rates were adjusted for hospital type and clustering within hospital site; slope changes are presented as incidence rate ratios (IRRs) with 95\% confidence intervals (Cls).

Results: In hospitals that had ceased screening $(n=13)$, there was an increase in slope after screening and isolation were discontinued compared with before screening and isolation were discontinued (slope change IRR 1.25 [95\% Cl 1.01-1.54]). In hospitals that continued screening $(n=50)$, the slope was not significantly different after June 2012 compared with before June 2012 (slope change IRR 0.81 [95\% Cl 0.56-1.15]).

Interpretation: There was a significant increase in the rate of rise of VRE-positive blood cultures in hospitals that discontinued active VRE screening and isolation programs but not in hospitals that continued to screen and isolate. Hospitals aiming to minimize rising rates should consider maintaining active screening and isolation programs.

V ancomycin-resistant Enterococcus (VRE) is an important nosocomial pathogen. ${ }^{1}$ Since its emergence in the $1980 \mathrm{~s},{ }^{2,3}$ rates of colonization and infection with this microorganism have risen dramatically in hospitals worldwide. ${ }^{4-8}$ Patients with bacteremia due to VRE are thought to have worse outcomes than patients with vancomycinsusceptible enterococcal bacteremia, including increased mortality and length of hospital stay. ${ }^{9}$ Preventing nosocomial VRE infections is therefore a patient safety priority in many jurisdictions. ${ }^{10-13}$

In an effort to minimize the spread of antimicrobial resistance within hospitals, it is widely recommended to emphasize hand hygiene, environmental cleaning and antimicrobial stewardship (including control of vancomycin use). ${ }^{14-18}$ Additional infection control interventions such as an active screen- ing program for patients at increased risk for colonization with VRE and placing patients colonized or infected with the organism on contact precautions have generated controversy owing to the lack of robust evidence. ${ }^{19,20}$ Active screening programs increase the identification of patients colonized with

Competing interests: Dr. Garber reports grants from Gilead outside the submitted work. Dr. Brown reports grants from AstraZeneca outside the submitted work. No other competing interests were declared.

This article has been peer reviewed.

Correspondence to: Jennie Johnstone, jennie.johnstone @ oahpp.ca CMAJ Open 2017. DOI:10.9778/cmajo.20160121 
VRE and reduce the time before contact precautions are implemented. ${ }^{21}$ However, the efficacy of contact precautions to prevent the spread of antimicrobial-resistant organisms including VRE has been questioned in 2 randomized controlled trials, ${ }^{22,23}$ and studies investigating the impact of discontinuation of VRE screening and isolation practices have given conflicting results. ${ }^{24-27}$

Infection control practices for VRE in Canadian institutions are increasingly heterogeneous, and there is no clear consensus on the best approach. ${ }^{28-30}$ In Ontario, before June 2012, all inpatient hospitals maintained active VRE screening and isolation programs; ${ }^{14}$ in June 2012, some hospitals discontinued this practice, citing concern over the effectiveness of the programs and potential adverse effects of isolating patients. ${ }^{31}$ The objective of this study was to examine the rates of VRE-positive blood cultures in Ontario between January 2009 and June 2015, and to determine whether discontinuation of active screening and isolation programs for this organism was associated with a change in the rate of rise of VREpositive blood cultures.

\section{Methods}

\section{Setting}

All 219 inpatient hospitals in Ontario publicly report their cases of VRE-positive blood cultures. Hospital facilities that reported at least 1 such validated case between Jan. 1, 2009 and June 30, 2015 were included for analysis as the final sample in our study. We classified hospitals as acute teaching, large community, small community, and complex continuing care and rehabilitation hospitals using Canadian Institute for Health Information ${ }^{32}$ definitions.

\section{Definition of VRE bacteremia}

We used the Ontario Ministry of Health and Long-Term Care's case definition for bacteremia due to VRE. ${ }^{33}$ Briefly, a case is defined as a patient identified with a laboratoryconfirmed bloodstream infection with VRE. A bloodstream infection is a single blood culture positive for strains of $E$. faecium or E. faecalis that have a minimum inhibitory concentration to vancomycin of $32 \mu \mathrm{g} / \mathrm{mL}$ or greater.

\section{Data sources}

Since January 2009, Ontario hospitals have been mandated to report cases of VRE bacteremia to the Ontario Ministry of Health and Long-Term Care via the Self-Reporting Initiative. Cases are reported publicly on the Health Quality Ontario website. ${ }^{10}$ We used these Health Quality Ontario data as the data source for this study. To minimize any potential false-positive cases in the data set, we validated the data by contacting infection control practitioners at each hospital site after each reporting quarter to confirm whether the reported cases met the case definition. To quantify the accuracy of the validated case count, we compared the hospital-verified count of cases of VRE-positive blood cultures with the number of VRE-positive blood cultures reported by hospital laboratories on quarterly surveys issued by the Insti- tute for Quality Management in Healthcare, during the same study period.

\section{Hospital VRE screening and isolation practices}

To identify whether an Ontario hospital ceased or continued VRE screening and isolation practices, we conducted a short survey in 2013, 2014 and 2015. The survey was sent to the infection control practitioners at each hospital site across the province (Appendix 1, www.cmajopen.ca/content/5/2/E273/ suppl/DC1). Follow-up reminder emails were sent to nonresponders at 3-week intervals. We contacted any remaining nonresponding sites by telephone until we achieved a $100 \%$ response rate. ${ }^{28}$

\section{Statistical analysis}

The study period consisted of 26 time-variable reporting quarters between Jan. 1, 2009 and June 30, 2015. The primary outcome was the slope change in the incidence rate of VREpositive blood cultures before versus after the intervention. All data were reported as the number of VRE-positive blood cultures divided by patient-days per quarter. Patient-days were provided by the Ontario Ministry of Health and LongTerm Care.

First, we used a slope term within a time series Poisson regression to assess the annual change in the incidence rate of VRE-positive blood cultures during the study period, as an incidence rate ratio (IRR). As such, a slope of 1.1 would represent a $10 \%$ multiplicative growth in the incidence rate per year, and a slope of 0.9 would represent a $10 \%$ reduction in incidence per year. Next, we stratified the cohort into 2 groups: a ceased-screening cohort, defined as hospitals that discontinued VRE screening and isolation practices at some point within the study period, and a screening cohort (used as a comparison group), which included hospitals that continued to screen and isolate patients throughout the study period. For the ceased-screening cohort, we defined the intervention date as the calendar quarter in which the hospital stopped screening and isolating for VRE. For the screening cohort, the intervention date was defined as beginning after June 2012 (quarter 15). We used an interrupted time series Poisson regression stratified by VRE control strategy (i.e., ceased or continued screening) to determine whether the slope of VRE-positive blood culture incidence rates was different after versus before the change in screening practice (slope change). For both cohorts, we examined the slope change by fitting an interaction between the intervention and time in the Poisson model (level and slope change model ${ }^{34}$ ). As with the slope, the slope change was reported as an IRR. The number of cases was used as the outcome, and the log of the number of patient-days was used as the offset. We adjusted the model for hospital type (acute teaching v. community), and we accounted for clustering within hospital site using a generalized estimating equation with the independence covariance structure. The unadjusted and adjusted slope change annual IRRs are presented alongside their associated $95 \%$ confidence intervals (CIs). We checked for the 
presence of residual autocorrelation within hospitals using a plot of the autocorrelation function.

We performed 3 additional sensitivity analyses. The first restricted the interrupted time series Poisson regression in the ceased-screening and screening cohorts to acute teaching hospitals, to better compare hospitals with similar patient acuity and case-mix. We defined acute teaching hospitals as acute and pediatric hospitals that provide highly complex patient care, are affiliated with a medical or health sciences school and have substantial research activity and postgraduate training. ${ }^{32}$ Second, to help maximize confidence in our analyses, we restricted the analyses to cases attributable to the reporting facility, given that these cases were more likely to have been acquired locally (when reporting a case of VRE-positive blood culture, hospital facilities are required to indicate whether the case is attributable to the reporting facility itself [i.e., symptom onset arising $>72 \mathrm{hr}$ after admission to the facility] or to another health care facility ${ }^{33}$ ). Last, we examined the main analyses and 2 sensitivity analyses for lagged intervention effects; follow-up times of 3 and 6 months following the intervention were excluded. We hypothesized that this exclusion should magnify any differences seen in the ceased-screening cohort analysis, as the impact of discontinuing screening and isolation practices, if present, would become more apparent over time (e.g., as colonization spread) but should have no effect in the screening cohort analysis.

For all analyses, a 2-tailed $p$ value of $<0.05$ was deemed as significant. We used SAS version 9.3 (SAS Institute) and $\mathrm{R}$ version 3.3.1 to analyze the data.

\section{Ethics approval}

The study received Research Ethics Board approval at Public Health Ontario before its commencement.

\section{Results}

In total, 525 VRE-positive blood cultures were reported publicly by hospitals during the study period (Figure 1). Contact with the hospitals showed that 130 cases $(24.8 \%)$ had been erroneously reported, resulting in 395 VRE-positive blood cultures as our final study sample. Reasons for erroneous reporting included reporting of a VRE-positive screening rectal swab rather than blood culture (82 cases) and missing records or data entry error (28); in 20 cases the reason was unknown. The final study sample was comparable to the number of cases reported to the Institute for Quality Management in Healthcare during the same period $(n=362)$.

Most VRE-positive blood cultures occurred in acute teaching hospitals (288 [72.9\%]), and over three-quarters (309 [78.0\%]) were attributable to the reporting facility About half of the cases (195 [49.4\%]) occurred in the ceased-screening cohort.

\section{Hospital screening and isolation practices}

The 395 VRE-positive blood cultures were reported by 63/219 (28.8\%) hospitals. All 63 hospitals responded to the VRE survey each year. Thirteen of the 63 hospitals discontinued VRE screening and isolation at some point during the study period: 9 acute teaching hospitals stopped in June 2012 (reporting quarter 15), 1 large community hospital in February

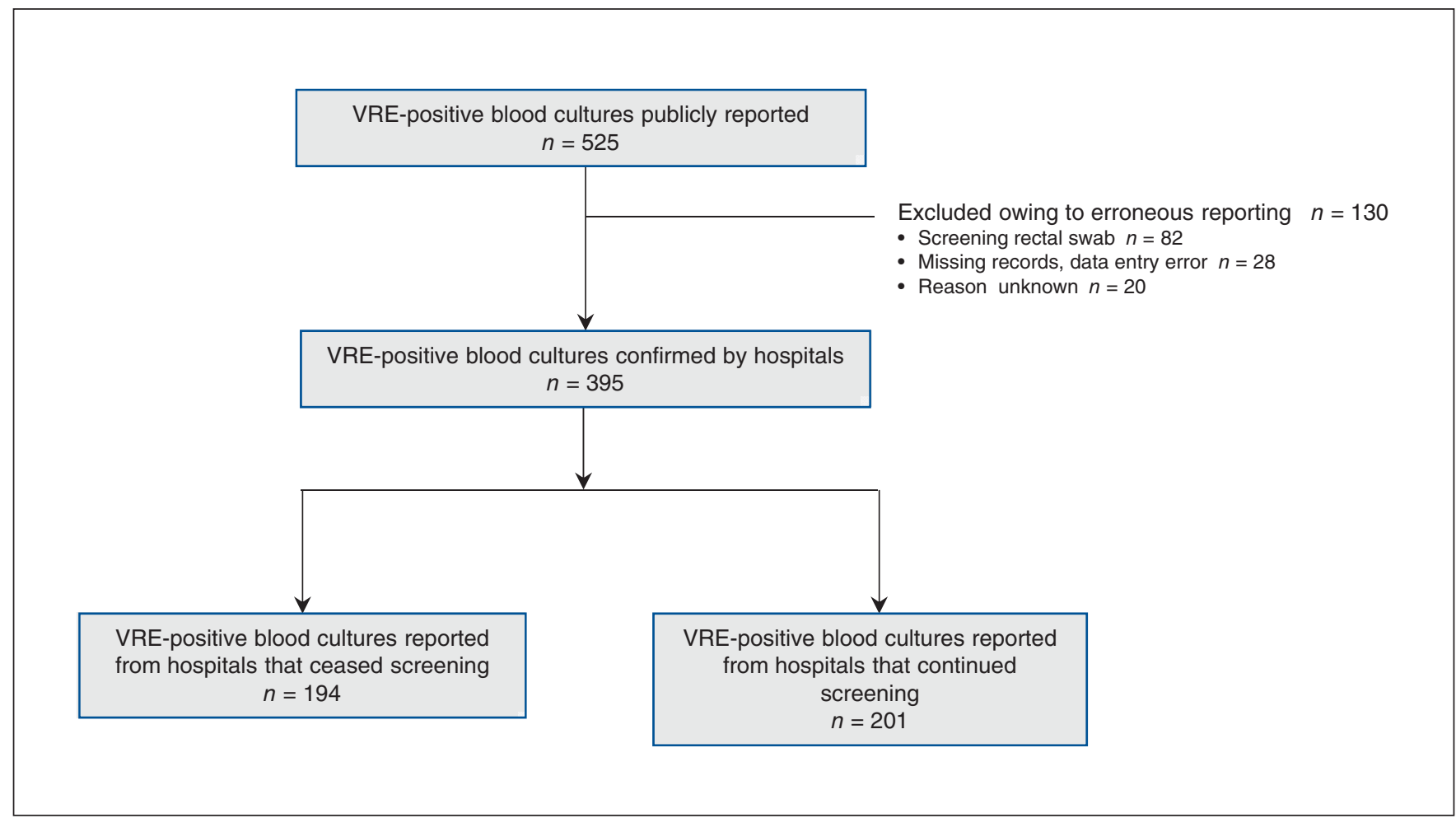

Figure 1: Flow diagram of blood cultures positive for vancomycin-resistant Enterococcus (VRE) occurring between January 2009 and June 2015 in Ontario. 


\section{OPEN}

\begin{tabular}{|c|c|c|c|}
\hline \multicolumn{4}{|c|}{$\begin{array}{l}\text { Table 1: Incidence rate of VRE-positive blood cultures per } \\
100000 \text { patient-days in Ontario between January } 2009 \text { and } \\
\text { June } 2015 \text {, overall and by whether VRE screening and } \\
\text { isolation practices were ceased or continued }\end{array}$} \\
\hline \multirow[b]{2}{*}{ Variable } & \multicolumn{3}{|c|}{ Incidence rate per 100000 patient-days } \\
\hline & $\begin{array}{c}\text { Total } \\
n=395\end{array}$ & $\begin{array}{l}\text { Screening } \\
\text { ceased } \\
n=194\end{array}$ & $\begin{array}{l}\text { Screening } \\
\text { continued } \\
n=201\end{array}$ \\
\hline \multicolumn{4}{|l|}{ Hospital type } \\
\hline Acute teaching & 1.74 & 2.50 & 1.11 \\
\hline Large community & 0.47 & 0.41 & 0.48 \\
\hline Small community & 1.81 & 0.88 & 2.13 \\
\hline \multicolumn{4}{|l|}{ Case attribution } \\
\hline Reporting facility & 0.81 & 1.70 & 0.54 \\
\hline Other facility & 0.07 & 0.07 & 0.06 \\
\hline Unknown & 0.16 & 0.36 & 0.09 \\
\hline \multicolumn{4}{|l|}{ Year } \\
\hline 2009 & 0.94 & 2.54 & 0.43 \\
\hline 2010 & 0.55 & 1.38 & 0.30 \\
\hline 2011 & 0.82 & 1.67 & 0.56 \\
\hline 2012 & 1.13 & 1.75 & 0.94 \\
\hline 2013 & 1.21 & 2.74 & 0.74 \\
\hline 2014 & 1.36 & 2.48 & 1.00 \\
\hline 2015 & 1.37 & 2.60 & 0.97 \\
\hline
\end{tabular}

2015 (reporting quarter 25), and 3 (2 large community hospitals and 1 small community hospital) in April 2015 (reporting quarter 26). The 50 remaining hospitals (10 acute teaching, 35 large community and 5 small community) continued to screen and isolate patients colonized or infected with VRE.

\section{Rates of VRE-positive blood culture}

When all blood cultures in the data set validated to be VREpositive were included ( $n=395$ cases), the overall rate during the study period was 1.04 per 100000 patient-days. The overall incidence rates of VRE-positive blood cultures per 100000 patient-days, overall and by cessation/continuation of VRE screening and isolation, are shown in Table 1 . The rate increased from 0.93 per 100000 patient-days in the first reporting quarter to 1.48 per 100000 patient-days in the last reporting quarter. The slope was statistically significant, with rates increasing by $12.5 \%$ per year (95\% CI 6.1-18.3) $(p<0.001)$ (Figure 2).

\section{Interrupted time series Poisson regression analysis}

In the ceased-screening cohort, in unadjusted analysis, there was a change in the slope after screening and isolation practices were discontinued compared to before the practices were discontinued (slope change IRR 1.37 [95\% CI 1.04-1.80]) ( $p=$ 0.03 ); adjusted analysis was similar (slope change IRR 1.25 (95\% CI 1.01-1.54]) ( $p=0.04$ ) (Table 2, Figure 3). In the screening group, in unadjusted analysis comparing rates after versus before June 2012, the slope change was not significantly different (IRR 0.80 [95\% CI 0.59-1.09]) ( $p=0.16)$. In adjusted analysis, the results were essentially the same (slope change IRR 0.81 [95\% CI 0.56-1.15]) ( $p=0.24$ ) (Table 2, Figure 3). The

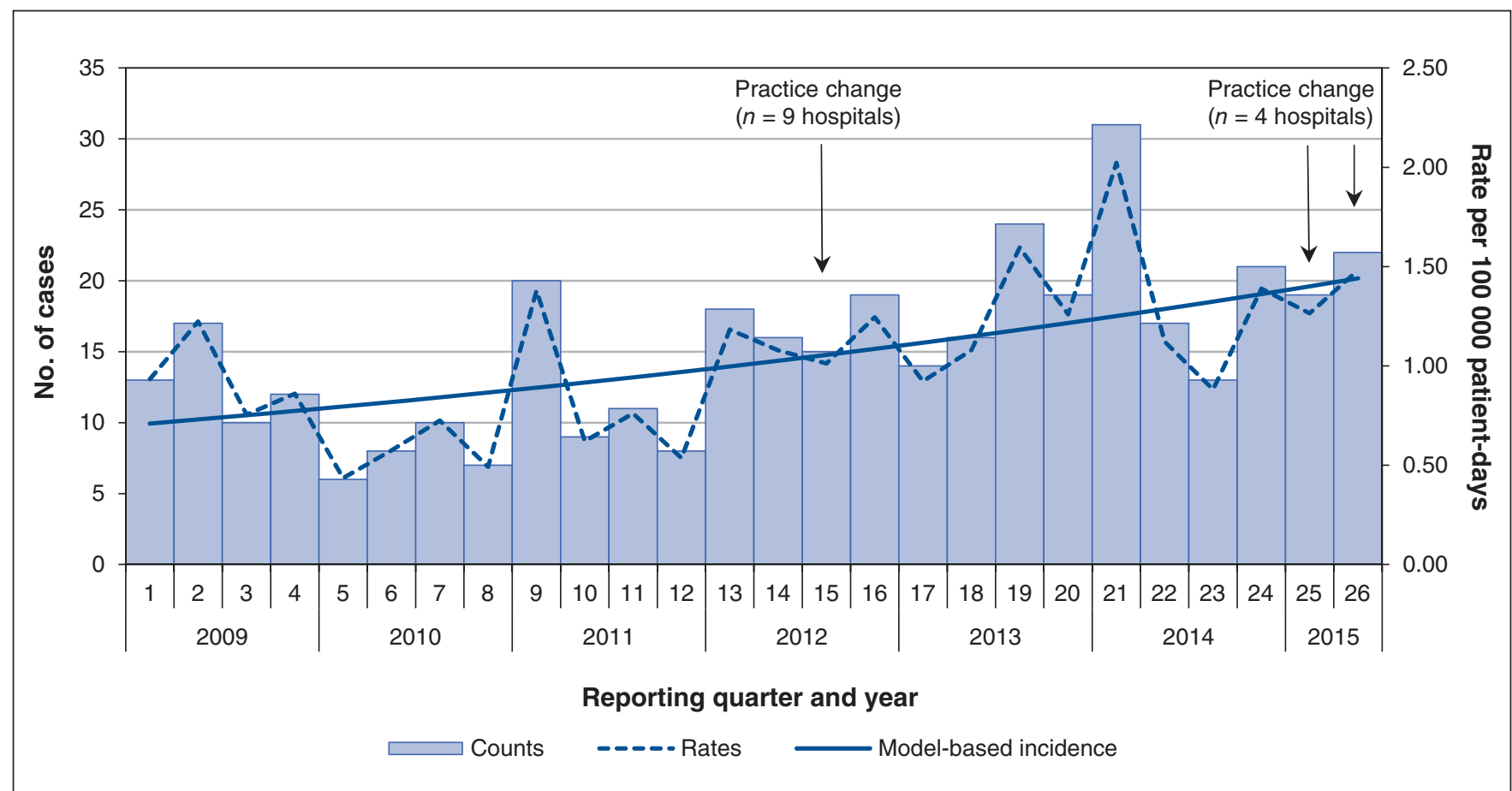

Figure 2: Numbers of cases and rates per 100000 patient-days of blood cultures positive for vancomycin-resistant Enterococcus, January 2009-June 2015. 
results of the sensitivity analyses restricting the analyses to acute teaching hospitals (9 in the ceased-screening cohort and 10 in the screening cohort) were essentially identical to the main analysis (Table 2, Figure 4), as were the results when the analyses were restricted to cases attributable to the reporting facility (Table 2). When lagged effects were incorporated into the model, the results were magnified for the ceased-screening cohort but remained unchanged for the screening cohort (Table 2). For both the screening and ceased-screening models, residual autocorrelation within each facility was low $(\rho<0.2)$.

\section{Interpretation}

In Ontario hospitals, overall rates of VRE-positive blood cultures almost doubled between January 2009 and June 2015. In hospitals that ceased screening and isolation programs, there was a significant increase in the rate of rise of VRE-positive blood cultures, and this was not seen in hospitals that continued to screen and isolate.

Our finding that discontinuation of active VRE screening and isolation of patients with VRE colonization or infection

\section{Table 2: Annual change in the incidence rate (slope*) of VRE-positive blood cultures before, after and slope change $†$ after versus before discontinuation of VRE screening and isolation (in ceased-screening cohort) and June 2012 (in screening cohort) in unadjusted and adjusted analysis and sensitivity analyses restricting analyses to 1) acute teaching hospitals, 2) cases attributable to the reporting facility and 3) lagged-effect models incorporating a 3-month lag and 6-month lag}

\begin{tabular}{|c|c|c|c|c|c|c|c|c|}
\hline \multirow[b]{2}{*}{ Variable } & \multicolumn{3}{|c|}{ Unadjusted incidence rate ratio } & \multirow{2}{*}{$\begin{array}{l}\text { Slope } \\
\text { change } \\
p \text { value }\end{array}$} & \multicolumn{3}{|c|}{ Adjusted incidence rate ratio } & \multirow{2}{*}{$\begin{array}{l}\text { Slope } \\
\text { change } \\
p \text { value }\end{array}$} \\
\hline & $\begin{array}{l}\text { Slope } \\
\text { before }\end{array}$ & $\begin{array}{l}\text { Slope } \\
\text { after }\end{array}$ & $\begin{array}{l}\text { Slope change } \\
\qquad(95 \% \mathrm{Cl})\end{array}$ & & $\begin{array}{l}\text { Slope } \\
\text { before }\end{array}$ & $\begin{array}{l}\text { Slope } \\
\text { after }\end{array}$ & $\begin{array}{l}\text { Slope change } \\
\qquad(95 \% \mathrm{Cl})\end{array}$ & \\
\hline \multicolumn{9}{|c|}{ Main analysis ( $n=395$ cases) } \\
\hline \multicolumn{9}{|c|}{ Ceased-screening cohort } \\
\hline No lag & 0.80 & 1.09 & $1.37(1.04-1.80)$ & 0.03 & 0.90 & 1.12 & $1.25(1.01-1.54)$ & 0.04 \\
\hline 3-mo lag & 0.80 & 1.20 & $1.51(1.14-2.00)$ & 0.004 & 0.90 & 1.24 & $1.38(1.07-1.79)$ & 0.01 \\
\hline 6-mo lag & 0.80 & 1.35 & $1.70(1.27-2.27)$ & 0.0004 & 0.90 & 1.39 & $1.56(1.19-2.03)$ & 0.001 \\
\hline \multicolumn{9}{|c|}{ Screening cohort } \\
\hline No lag & 1.32 & 1.06 & $0.80(0.59-1.09)$ & 0.2 & 1.31 & 1.06 & $0.81(0.56-1.15)$ & 0.2 \\
\hline 3-mo lag & 1.32 & 1.23 & $0.93(0.68-1.28)$ & 0.7 & 1.31 & 1.23 & $0.94(0.66-1.33)$ & 0.7 \\
\hline 6-mo lag & 1.32 & 1.52 & $1.15(0.83-1.60)$ & 0.4 & 1.31 & 1.52 & $1.16(0.81-1.66)$ & 0.4 \\
\hline \multicolumn{9}{|c|}{ Acute teaching hospitals ( $n=287$ cases) } \\
\hline \multicolumn{9}{|c|}{ Ceased-screening cohort } \\
\hline No lag & 0.84 & 1.12 & $1.34(0.99-1.83)$ & 0.06 & 0.84 & 1.13 & $1.35(1.13-1.60)$ & $<0.01$ \\
\hline 3-mo lag & 0.84 & 1.25 & $1.49(1.09-2.05)$ & 0.01 & 0.84 & 1.25 & $1.49(1.19-1.88)$ & $<0.01$ \\
\hline 6-mo lag & 0.84 & 1.41 & $1.68(1.22-2.32)$ & 0.002 & 0.84 & 1.41 & $1.68(1.32-2.14)$ & $<0.001$ \\
\hline \multicolumn{9}{|c|}{ Screening cohort } \\
\hline No lag & 1.23 & 0.88 & $0.72(0.46-1.13)$ & 0.2 & 1.23 & 0.88 & $0.72(0.45-1.15)$ & 0.2 \\
\hline 3-mo lag & 1.23 & 1.01 & $0.82(0.52-1.30)$ & 0.4 & 1.23 & 1.01 & $0.82(0.53-1.27)$ & 0.4 \\
\hline 6-mo lag & 1.23 & 1.40 & $1.13(0.70-1.83)$ & 0.6 & 1.23 & 1.40 & $1.13(0.98-1.88)$ & 0.6 \\
\hline \multicolumn{9}{|c|}{ Cases attributable to reporting facility ( $n=309$ cases) } \\
\hline \multicolumn{9}{|c|}{ Ceased-screening cohort } \\
\hline No lag & 0.86 & 1.16 & $1.35(0.99-1.85)$ & 0.06 & 0.93 & 1.18 & $1.27(0.95-1.70)$ & 0.1 \\
\hline 3-mo lag & 0.86 & 1.30 & $1.51(1.09-2.08)$ & 0.01 & 0.93 & 1.32 & $1.42(0.96-2.10)$ & 0.08 \\
\hline 6-mo lag & 0.86 & 1.40 & $1.62(1.17-2.26)$ & 0.004 & 0.93 & 1.42 & $1.53(1.05-2.23)$ & 0.03 \\
\hline \multicolumn{9}{|c|}{ Screening cohort } \\
\hline No lag & 1.47 & 1.00 & $0.68(0.48-0.97)$ & 0.03 & 1.46 & 1.00 & $0.69(0.48-0.98)$ & 0.04 \\
\hline 3-mo lag & 1.47 & 1.14 & $0.77(0.54-1.11)$ & 0.2 & 1.46 & 1.14 & $0.78(0.55-1.11)$ & 0.2 \\
\hline 6-mo lag & 1.47 & 1.41 & $0.96(0.66-1.39)$ & 0.8 & 1.46 & 1.41 & $0.96(0.67-1.39)$ & 0.8 \\
\hline $\begin{array}{l}\text { Note: } \mathrm{Cl}=\text { confi } \\
\text { *Defined as the } \\
\text { of } 0.9 \text { would rep } \\
\text { tDefined as the } \\
2012 \text { v. before } J\end{array}$ & $\begin{array}{l}\text { slope af } \\
\text { the scre }\end{array}$ & $\begin{array}{l}\text { essation } \\
\text { hort). }\end{array}$ & $\begin{array}{l}\text { Enterococcus. } \\
\text { or example, a slope of } \\
\text { ar. } \\
\text { eening divided by the }\end{array}$ & $\begin{array}{l}\text { ould repres } \\
\text { before the }\end{array}$ & $\begin{array}{l}0 \% \text { multip } \\
\text { on of scre }\end{array}$ & $\begin{array}{l}\text { growth } \\
\text { the ce }\end{array}$ & $\begin{array}{l}\text { incidence rate per ye } \\
\text { screening cohort (and }\end{array}$ & $\begin{array}{l}\text { nd a slope } \\
\text { r June }\end{array}$ \\
\hline
\end{tabular}




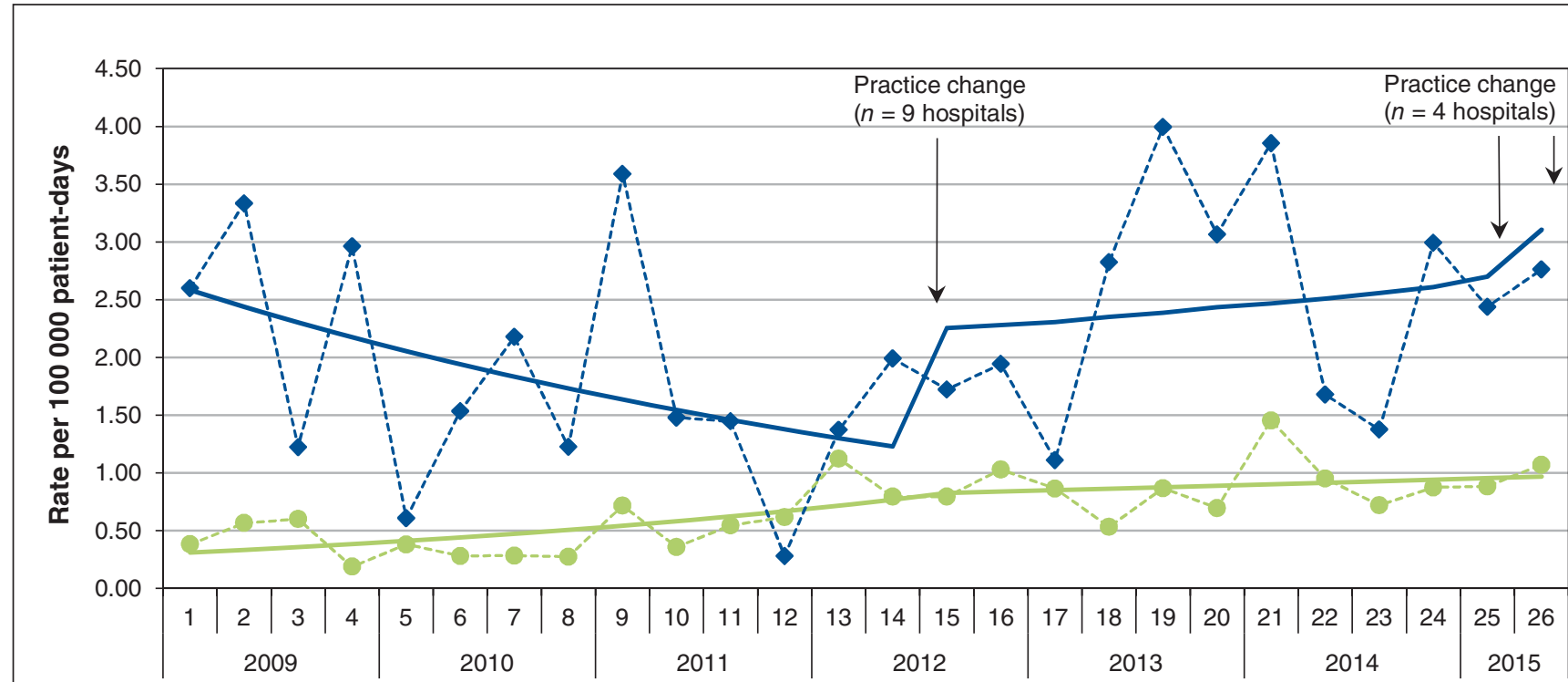

Reporting quarter and year

Hospitals that ceased screening (model-based)

Hospitals that continued screening (model-based)

-.- Hospitals that ceased screening (observed)

-.-- Hospitals that continued screening (observed)

Figure 3: Rates of blood cultures positive for vancomycin-resistant Enterococcus (VRE), January 2009-June 2015, stratified by hospitals that ceased VRE screening and isolation practices within the study period versus hospitals that continued screening and isolation practices within the study period.

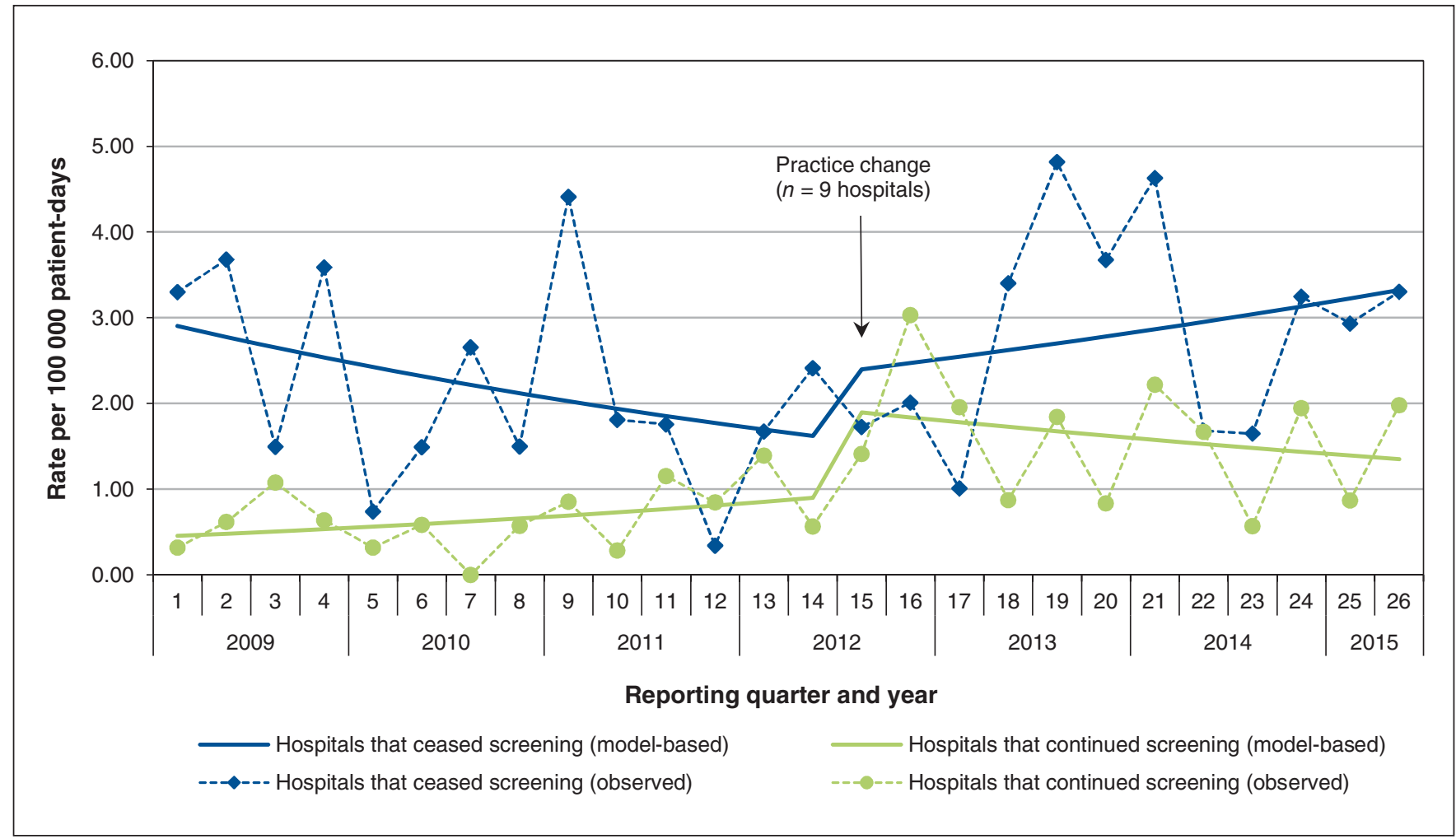

Figure 4: Rates of blood cultures positive for vancomycin-resistant Enterococcus (VRE) in acute teaching hospitals, January 2009-June 2015, stratified by hospitals that ceased VRE screening and isolation practices within the study period $(n=9)$ versus hospitals that continued screening and isolation practices within the study period $(n=10)$. 
was associated with an increased rate of rise of VRE-positive blood cultures is consistent with several prior observational studies, which similarly suggest that active screening for VRE colonization and isolation of those affected is associated with reduced rates of bacteremia due to VRE. ${ }^{24,26,35}$ In contrast, our results are not consistent with 2 recent studies. ${ }^{27,31}$ However, one study ${ }^{27}$ was conducted in an immunocompromised patient population at a single centre where all patients were admitted to single-bed rooms with private bathroom. Thus, the results may not be generalizable to less specialized hospital settings where patients are admitted to multipatient rooms and share bathrooms. A second observational study ${ }^{31}$ included some of the data from the ceased-screening hospital cohort in our study and showed a trend toward increased VRE-positive blood cultures, but the trend did not reach statistical significance. The difference in findings might be explained by the shorter duration of follow-up in that study (18 mo v. 36 mo in our study) and, consequently, fewer data points.

There have been 2 cluster randomized controlled trials investigating the efficacy of screening for antimicrobialresistant organisms and isolating affected patients in the intensive care unit setting. ${ }^{22,23}$ The results of those trials raise questions about the use of contact precautions for preventing transmission of antibiotic-resistant organisms; however, neither trial reported on the results of VRE bacteremia, and neither was powered to detect this difference. ${ }^{22,23}$ Thus, no randomized controlled trial data are available that definitively answer whether discontinuing screening and isolation for $\mathrm{VRE}$ is associated with increased rates of bacteremia due to this organism.

\section{Strengths and limitations}

Our study has many strengths, including comprehensive data collection from multiple hospitals over a 6-year period encompassing approximately 38000000 patient-days and a quasi-experimental study design using an interrupted time series Poisson regression model with an objective outcome. However, several limitations require consideration. First, we did not have data on potential confounders such as changes over time in adherence to hand hygiene guidelines, environmental services activities and antibiotic use within each hospital. We included a comparison cohort and would have expected changes in these potential confounders to apply to this group as well, particularly when comparing acute teaching hospitals. Second, this study had a quasi-experimental design and was not a randomized controlled trial and was therefore susceptible to biases inherent to this type of design, including regression to the mean as a potential explanation for the results. To help mitigate this potential bias, our sensitivity analyses incorporated lag effects, which amplified the results in the ceased-screening cohort, thus increasing the level of confidence in our results. Third, we did not have patient-level clinical data, and the clinical consequences of having a VREpositive blood culture were unknown; we chose to use the term "VRE-positive blood culture" rather than "VRE bacteremia" because of this limitation. Last, misclassification bias may have occurred as we did not have knowledge of patients' prior hospital stays; thus, a patient might have been colonized with VRE in a ceased-screening hospital but had the positive blood culture in a screening hospital and vice versa. We included a sensitivity analysis that restricted the analyses to positive blood cultures attributable only to the reporting facility to mitigate this potential bias as these cases were more likely to have been acquired locally. However, the attribution is only for the positive blood culture and not necessarily for the acquisition of VRE colonization, and the possibility of misclassification remains. Additional misclassification may have occurred owing to false-positive and false-negative culture results in the data set. We sought to minimize this bias by validating each reported positive case with the individual hospital and verifying case count accuracy with a separate data set. The large number of false-positive results in the original data set $(25 \%)$ was unexpected and highlights the importance of validation when using publicly reported data as well as the need for enhanced education for those imputing data at the local level.

\section{Conclusion}

Whether there is still a need to prevent transmission of VRE, given the availability of effective anti-VRE therapy, has been debated; however, the best available evidence suggests that patients with VRE-positive blood cultures have an associated increased risk of death and longer duration of hospital stay compared to patients with vancomycin-susceptible blood cultures, and the emergence of daptomycin and linezolid resistance remains a concern. Future research should better characterize the costs and benefits of screening and isolation programs. In summary, rates of VRE-positive blood cultures have increased in Ontario. Hospitals aiming to minimize the rising rate of VRE-positive blood cultures should consider maintaining active screening and isolation programs.

\section{References}

1. Murray BE. Vancomycin-resistant enterococcal infections. N Engl 7 Med 2000; 342:710-21.

2. Leclercq R, Derlot E, Duval J, et al. Plasmid-mediated resistance to vancomycin and teicoplanin in Enterococcus faecium. N Engl 7 Med 1988;319:157-61.

3. Uttley AH, Collins $\mathrm{CH}$, Naidoo J, et al. Vancomycin-resistant enterococci. Lancet 1988;1:57-8.

4. Vancomycin-resistant enterococci infections in Canadian acute-care hospitals: surveillance report Fanuary 1, 1999 to December 31, 2011. Ottawa: Centre for Communicable Diseases and Infection Control, Public Health Agency of Canada; 2013.

5. Adam HJ, DeCorby M, Rennie R, et al.; Canadian Antimicrobial Resistance Alliance (CARA). Prevalence of antimicrobial resistant pathogens from blood cultures from Canadian hospitals: results of the CANWARD 2007-2009 study. Diagn Microbiol Infect Dis 2011;69:307-13.

6. Bourdon N, Fines-Guyon M, Thiolet JM, et al. Changing trends in vancomycin-resistant enterococci in French hospitals, 2001-08. 7 Antimicrob Chemother 2011;66:713-21.

7. Gastmeier P, Schröder C, Behnke M, et al. Dramatic increase in vancomyinresistant enterococci in Germany. 7 Antimicrob Chemother 2014;69:1660-4.

8. National Nosocomial Infections Surveillance System. National Nosocomial Infections Surveillance (NNIS) System Report, data summary from January 1992 through June 2004, issued October 2004. Am 7 Infect Control 2004;32: 470-85.

9. Prematunge C, MacDougall C, Johnstone J, et al. VRE and VSE bacteremia outcomes in the era of effective VRE therapy: a systematic review and metaanalysis. Infect Control Hosp Epidemiol 2016;37:26-35.

10. Patient safety individual results — introduction. Toronto: Health Quality Ontario; 2016. Available: www.hqontario.ca/portals/0/Modals/pr/patient -safety/en/individual/index-en.html (accessed 2016 July 12).

11. Key projects on patient safety. Ottawa: Canadian Institute for Health Information; 2016. Available: https://www.cihi.ca/en/health-system-performance/ 
quality-of-care-and-outcomes/patient-safety/key-projects-on-patient-safety (accessed 2016 July 12).

12. Vancomycin-resistant enterococci (VRE). Ottawa: Canadian Patient Safety Institute; 2016. Available: www.patientsafetyinstitute.ca/en/Topic/Pages/ Vancomycin-resistant-Enterococci-(VRE).aspx (accessed 2016 July 12).

13. Antibiotic resistance threats in the United States, 2013. Atlanta: Centers for Disease Control and Prevention; 2013. Available: www.cdc.gov/drugresistance/ threat-report-2013/pdf/ar-threats-2013-508.pdf\#page=5 (accessed 2016 July 13).

14. Ontario Agency for Health Protection and Promotion, Provincial Infectious Diseases Advisory Committee. Annex A-screening, testing and surveillance for antibiotic-resistant organisms (AROs). Annexed to: Routine practices and additional precautions in all health care settings. Toronto: Queen's Printer for Ontario; 2013.

15. Weiner LM, Fridkin SK, Aponte-Torres Z, et al. Vital signs: preventing antibiotic-resistant infections in hospitals - United States, 2014. MMWR Morb Mortal Wkly Rep 2016;65:235-41.

16. Cookson BD, Macrae MB, Barrett SP, et al.; Combined Working Party of the Hospital Infection Society and Infection Control Nurses Association. Guidelines for the control of glycopeptide-resistant enterococci in hospitals. 7 Hosp Infect 2006;62:6-21.

17. Nicolle LE. Infection control programmes to contain antimicrobial resistance. Geneva: World Health Organization; 2001.

18. Muto CA, Jernigan JA, Ostrowsky BE, et al.; SHEA. SHEA guideline for preventing nosocomial transmission of multidrug-resistant strains of Staphylococcus aureus and Enterococcus. Infect Control Hosp Epidemiol 2003;24:362-86.

19. Ho C, Lau A, Cimon K, et al. Screening, isolation, and decolonization strategies for vancomycin-resistant enterococci or extended spectrum beta-lactamase producing organisms: a systematic review of the clinical evidence and health services impact [rapid response report: systematic review]. Ottawa: Canadian Agency for Drugs and Technologies in Health; 2012.

20. Talbot TR. Two studies feed the debate on active surveillance for methicillin-resistant Staphylococcus aureus and vancomycin-resistant enterococci carriage: To screen or not to screen? I Infect Dis 2007;195:314-7.

21. Huang SS, Rifas-Shiman SL, Pottinger JM, et al.; Centers for Disease Control and Prevention Epicenters Program. Improving the assessment of vancomycinresistant enterococci by routine screening. F Infect Dis 2007;195:339-46.

22. Huskins WC, Huckabee CM, O'Grady NP, et al. Intervention to reduce transmission of resistant bacteria in intensive care. N Engl 7 Med 2011;364:1407-18.

23. Derde LP, Cooper BS, Goossens H, et al.; MOSAR WP3 Study Team. Interventions to reduce colonisation and transmission of antimicrobialresistant bacteria in intensive care units: an interrupted time series study and cluster randomised trial. Lancet 2014;14:31-9.

24. Price CS, Paule S, Noskin GA, et al. Active surveillance reduces the incidence of vancomycin-resistant enterococcal bacteremia. Clin Infect Dis 2003;37:921-8.

25. Perencevich EN, Fisman DN, Lipsitch M, et al. Projected benefits of active surveillance for vancomycin-resistant enterococci in intensive care units. Clin Infect Dis 2004:38:1108-15.

26. Hachem R, Graviss L, Hanna H, et al. Impact of surveillance for vancomycinresistant enterococci on controlling a bloodstream outbreak among patients with hematologic malignancy. Infect Control Hosp Epidemiol 2004;25:391-4.

27. Almyroudis NG, Osawa R, Samonis G, et al. Discontinuation of systematic surveillance and contact precautions for vancomycin-resistant Enterococcus (VRE) and its impact on the incidence of VRE faecium bacteremia in patients with hematologic malignancies. Infect Control Hosp Epidemiol 2016;37:398-403.

28. Nadolny E, Prematunge C, Policarpio M, et al. Vancomycin-resistant Enterococcus (VRE) screening and isolation practices (SIP) scan: hospital-based infection control policy in Ontario (2012-2015). Proceedings of Wisdom Begins with Wonder: IPAC Canada 2016 Annual Conference; 2016 May 15-17; Niagara Falls $(O N)$. Winnipeg: Infection Prevention and Control Canada; 2016.

29. CHICA - Canada position statement - VRE screening and contact precautions [updated June 29, 2012]. Winnipeg: Infection Prevention and Control Canada; 2012. Available: https://ipac-canada.org/photos/custom/Members/ pdf/VRE\%20Position\%20Statement\%20-\%20FINAL.pdf (accessed 2017 Mar. 24).

30. Canadian Consensus Development Conference: surveillance and screening of AROs (antimicrobial-resistant organisms) - consensus statement of the jury. IHE Consensus Statements vol 6. Edmonton: Institute of Health Economics; 2014. Available: http://www.ihe.ca/download/consensus_statement_on_ surveillance_screening_for_aros_antimicrobial_resistant_organisms.pdf (accessed 2017 Mar. 24).

31. Lemieux C, Gardam M, Evans G, et al. Longitudinal multicenter analysis of outcomes after cessation of control measures for vancomycin-resistant enterococci. Infect Control Hosp Epidemiol 2017;38:24-30.

32. Hospital report 2007: acute care. Ottawa: Canadian Institute for Health Information; 2007. Available: https://secure.cihi.ca/free_products/OHA_Acute07 EN_final_secure.pdf (accessed 2016 July 13).

33. Vancomycin resistant Enterococcus (VRE bacteremia) infection rate. Resource for Indicator Standards (RIS). Toronto: Ontario Ministry of Health and Long-Term Care; 2012. Available: www.health.gov.on.ca/en/pro/programs/ ris/docs/vancomycin_resistant_enterococcus_infection_rate_en.pdf (accessed 2016 July 13).

34. Lopez Bernal J, Cummins S, Gasparrini A. Interrupted time series regression for the evaluation of public health interventions: a tutorial. Int 7 Epidemiol 2016 June 8 [Epub ahead of print]. doi:10.1093/ije/dyw098.

35. Mikulska M, Del Bono V, Raiola AM, et al. Enterococcal bloodstream infection after hematopoietic stem cell transplant: experience of a center with a low prevalence of vancomycin-resistant enterococci. Clin Infect Dis 2012;55:1744

Affiliations: Public Health Ontario (Johnstone, Policarpio, Lam, Adomako, Prematunge, Nadolny, Li, Brown, Garber); St. Joseph's Health Centre (Johnstone); Department of Medicine (Johnstone, Garber); Dalla Lana School of Public Health (Johnstone, Li, Brown), University of Toronto; Institute for Quality Management in Healthcare (Kerr), Toronto, Ont.; Department of Medicine (Garber), University of Ottawa, Ottawa, Ont.

Contributors: All of the authors contributed to the study design and execution, data analysis and interpretation and drafting and revising the manuscript, approved the final version to be published and agreed to act as guarantors of the work.

Acknowledgements: The authors acknowledge the following for their contributions to this project: Christine Fleming, Institute for Quality Management in Healthcare, for support with validating clinical isolates positive for vancomycin-resistant Enterococcus; Cathy Egan for her unwavering support of the project; and Lauren Churcher, Ontario Ministry of Health and Long-Term Care, for her valued partnership and support of this research project.

Supplemental information: For reviewer comments and the original submission of this manuscript, please see www.cmajopen.ca/content/5/2/ E273/suppl/DC1 